Bull. Austral. Math. Soc.

$41 \mathrm{~A} 28,41 \mathrm{~A} 30,41 \mathrm{~A} 65$

VOL. $40(1989) \quad[37-48]$

\title{
BOUNDED MEASURABLE SIMULTANEOUS MONOTONE APPROXIMATION
}

\author{
SALEM M.A. SAHAB
}

Let $X=[a, b]$ be a closed bounded real interval. Let $B$ be the closed linear space of all bounded real valued functions defined on $X$, and let $M \subset B$ be the closed convex. cone consisting of all monotone non-decreasing functions on $X$. For $f, g \in B$ and a fixed positive $w \in B$, we define the so-called best $L_{\infty}$-sinultaneous approximant of $f$ and $g$ to be an element $h^{*} \in M$ satisfying

$$
\max \left(\left\|f-h^{*}\right\|_{w},\left\|g-h^{*}\right\|_{w}\right)=d \leqslant \max \left(\|f-h\|_{w},\|g-h\|_{w}\right)
$$

for all $h \in M$, where

$$
\|f\|_{w}=\sup _{a \leqslant x \leqslant b} w(x)|f(x)| .
$$

We establish a duality result involving the value of $d$ in terms of $f, g$ and $w$ only. obtained.

If in addition $f, g$ and $w$ are continuous, then some characterisation results are

\section{INTRODUCTION}

Let $X=[a, b]$ be a closed bounded interval of the real line. Let $B=B(X)$ be the linear space of all bounded real valued functions defined on X. Let $M=M(X) \subseteq B$ be the closed convex cone of monotone non-decreasing functions defined on $X$. Given a fixed $w \in B, w(x) \geqslant \delta>0$ for all $x \in X$, define a weighted uniform norm $\|\cdot\|_{w}$ on $B$ by

$$
\|f\|_{w}=\sup (w(x)|f(x)|: x \in X) .
$$

The problem we are investigating in this paper is : Given $f$ and $g$ in $B$, find $h^{*} \in M$, if one exists, such that

$$
d=\max \left(\left\|f-h^{*}\right\|_{w},\left\|g-h^{*}\right\|_{w}\right)=\inf \max \left(\|f-h\|_{w},\|g-h\|_{w}\right) .
$$

where the infimum is taken over all $h$ in $M$. Such $h^{*}$ is called a best $L_{\infty}$-simultaneous approximant of $f$ and $g$, abbreviated b.s.a. . Note that if $f \neq g$ then $d>0$. Of course, when $w \equiv 1$, we have the usual well known uniform, or Tchebychev, norm.

Received 13 September 1988

Copyright Clearance Centre, Inc. Serial-fee code: 0004-9729/89 \$A2.00+0.00. 
In [1] Ublaya treated the case of the $L_{\infty}$-approximation to a single function $f$ by elements of $M$. He gave an explicit formula for computing $d$ in terms of $f$ and $w$ only, where $f$ is the function to be approximated with respect to the norm given by (1). He also characterised the set of all $L_{\infty}$ approximants of $f$, and he established properties of this solution set and its behaviour on some parts of $X$. In addition, if $f, w$ are continuous, $f \notin M$, he proved the existence of an infinitely differentiable function $h \in M$ which is a best $L_{\infty}$-approximant of $f$.

Our main objective here is to generalise Ubhaya's results to the simultaneous approximation case. In Section 2 we start with the elimination of the trivial possibilities of values of $d$ compared to the value of the distance between $M$ and either of $f$ or $g$ alone. Then we generalise the duality results established in [1]. We also show the existence of a function $h^{*} \in M$ satisfying (2), and we give an explicit expression of the set of all such solutions which clearly forms a convex subset of $M$.

For simplicity, we supress $w$ from the norm notation in (1) and (2).

\section{Duality and Characterisation}

Lemma 1. Suppose that $f, g \in B \cap M$. Then $h^{*}=(f+g) / 2$ is a best $L_{\infty}$ simultaneous approximant of $f$ and $g$.

Proof: Suppose there exists $h \in M$ such that

$$
\max (\|f-g\|,\|g-h\|)<\max \left(\left\|f-h^{*}\right\|,\left\|g-h^{*}\right\|\right)=\|f-g\| / 2 \text {. }
$$

Then

$$
\begin{aligned}
\|f-g\|=\|f-h+h-g\| & \leqslant\|f-h\|+\|g-h\| \\
& <\|f-g\| / 2+\|f-g\| / 2=\|f-g\| .
\end{aligned}
$$

This is a contradiction! This establishes the Lemma. However it can be easily seen by an example that $h^{*}$ is not unique in general.

Remark 1. (i) When $f=g$ we end up with the single approximation case discussed in [1].

(ii) If $f \neq g$, and there exists an element $f_{\infty} \in M$ such that $\left\|g-f_{\infty}\right\| \leqslant\left\|f-f_{\infty}\right\|$ and $f_{\infty}$ is a best $L_{\infty}$-approximant of $f$, then clearly

$$
\max \left(\left\|g-f_{\infty}\right\|,\left\|f-f_{\infty}\right\|\right)=\left\|f-f_{\infty}\right\| \leqslant\|f-h\| \leqslant \max (\|f-h\|,\|g-h\|)
$$

for all $h \in M$ and hence $f_{\infty}$ is a best $L_{\infty}$-simultaneuos approximant of $f$ and $g$.

To this end, we shall exclude for all practical purposes the three cases encountered above in Lemma 1 and Remark 1. With this assumption in mind we proceed to the next step. 
Let $\triangle$ be the closed triangle given by

$$
\triangle=\{(x, y) \in[a, b] \times[a, b]: x \leqslant y\}
$$

We also define the following

$$
\begin{aligned}
u(x, y) & =w(x) w(y) /(w(x)+w(y)) \\
\theta_{1} & =\sup \{u(x, y)(f(x)-g(y)):(x, y) \in \triangle\} \\
\theta_{2} & =\sup \{u(x, y)(g(x)-f(y)):(x, y) \in \triangle\} \\
\theta & =\max \left\{\theta_{1}, \theta_{2}\right\} \\
T_{1} & =\{(x, y) \in \triangle: u(x, y)(f(x)-g(y))=\theta\} \\
T_{2} & =\{(x, y) \in \triangle: u(x, y)(g(x)-f(y))=\theta\} \\
T & =T_{1} \cup T_{2} \\
P & =\bigcup\{[x, y]:(x, y) \in T\} \\
m(x, y) & =(w(x) f(x)+w(y) g(y)) /(w(x)+w(y)), \quad x, y \in X
\end{aligned}
$$

Finally define the functions $\underline{h}$ and $\bar{h}$ on $[a, b]$ by

$$
\begin{aligned}
& \underline{h}(x)=\sup \{[f(z) \vee g(z)-\theta / w(z)]: z \in[a, x]\} \\
& \bar{h}(x)=\inf \{[f(z) \wedge g(z)+\theta / w(z)]: z \in[x, b]\}
\end{aligned}
$$

where $f \vee g=\max (f, g)$ and $f \wedge g=\min (f, g)$.

Remark 2. (i) In general $\theta_{1} \neq \theta_{2}$. We assume here that $\theta_{2} \leq \theta_{1}=\theta$.

(ii) $T \neq \emptyset$. However $T$ might consist of a single point $(x, y)$ with $x \leq y$, hence $P$ could consist of a single point $x \in[a, b]$.

(iii) $\underline{h}$ and $\bar{h}$ are both monotone non-decreasing .

(iv) $\theta=0$ if and only if $f=g \in M$.

(v) If $h^{*}$ is a best $L_{\infty}$-simultaneous approximant of $f$ and $g$, then $h^{*}+c$ is a best $L_{\infty}$-simultaneous approximant of $f+c$ and $g+c$ where $c$ is a constant. Therefore we may assume without loss of generality that both $f$ and $g$ are non-negative and so is $h^{*}$.

Example. Let $X=[0,1]$. Define $f$ and $g$ as follows: $f(0)=3, f(1 / 3)=0, f(2 / 3)=$ $5, f(1)=4$ and the graph of $f$ is linear between these points. Let $g(0)=3, g(1 / 2)=1$, $g(2 / 3)=1, g(1)=3$ and the graph of $g$ is linear between these points. Let $w \equiv 1$. Then $\theta_{1}=(5-1) / 4=2>\theta_{2}=(3-0) / 2=3 / 2, T=\{(2 / 3,2 / 3)\}$ and $P=\{2 / 3\}$. Notice also that $\underline{h}(2 / 3)=\bar{h}(2 / 3)=m(2 / 3,2 / 3)=3$, and $\underline{h}(x)<\bar{h}(x)$ for all $x \neq 2 / 3$. However $\|f-\underline{h}\|=\|g-\underline{h}\|=\|f-\bar{h}\|=\|g-\bar{h}\|=2=\theta_{1}=\theta$. 
Remark 3. By [1], the $L_{\infty}$-distance between $f$ and $M$ is given by

$$
\theta_{f}=\sup _{(x, y) \in \triangle} u(x, y)(f(x)-f(y))
$$

Clearly $\theta \geqslant \max \left(\theta_{f}, \theta_{g}\right)$, because of the assumption following Remark 1 .

Theorem 2. Let $f, g$ and $w$ be as specified in Section 1. Let $\theta$ be as defined above. Then

$$
\theta=d=\inf _{h \in M} \max (\|f-h\|,\|g-h\|)
$$

Hence $\theta \leqslant \max (\|f\|,\|g\|)$.

Proof: We show first that $\theta=\theta_{1} \leqslant \eta=\max (\|f-h\|,\|g-h\|)$ for any arbitrary $h \in M$. So let $(x, y) \in \triangle$. Then

and

$$
\begin{aligned}
& w(x)|f(x)-h(x)| \leqslant\|f-h\| \leqslant \eta \\
& w(y)|g(y)-h(y)| \leqslant\|g-h\| \leqslant \eta .
\end{aligned}
$$

By monotonicity of $h$, we have $h(y)-h(x) \geqslant 0$, so we obtain

or

$$
\begin{aligned}
f(x)-g(y) \leqslant & f(x)-g(y)+h(y)-h(x) \\
\leqslant & (w(x)|f(x)-h(x)| / w(x))+(w(y)|g(y)-h(y)| / w(y)), \\
\leqslant & \|f-h\| / w(x)+\|g-h\| / w(y) \\
\leqslant & (1 / w(x)+1 / w(y)) \eta=(w(x)+w(y)) \eta / w(x) w(y), \\
& u(x, y)(f(x)-g(y)) \leqslant \eta .
\end{aligned}
$$

Since $(x, y) \in \triangle$ is arbitrary, we conclude that $\theta \leqslant \eta$. Since $h$ was arbitrary, we get $\theta \leqslant d$. Next we show that $\theta=\max (\|f-\underline{h}\|,\|g-\underline{h}\|)$. Let $x \in[a, b]$. By the definition of $\underline{h}$, we have $\underline{h}(x) \geqslant f(x) \vee g(x)-\theta / w(x)$, or equivalently

$$
w(x)(\underline{h}(x)-f(x)) \geqslant-\theta,
$$

and

$$
w(x)(\underline{h}(x)-g(x)) \geqslant-\theta .
$$

Now, let $\varepsilon>0$ be given. Then there exists $z \in[a, x]$ such that

$$
\underline{h}(x) \leqslant f(z) \vee g(z)-\theta / w(z)+\varepsilon .
$$

We have two symmetric cases to consider. It suffices to treat one of them: 
Case 1. $f(z) \geqslant g(z)$, so

$$
\underline{h}(x) \leqslant f(z)-\theta / w(z)+\varepsilon .
$$

By the definition of $\theta$ we have

or

$$
\begin{gathered}
\theta \geqslant(1 / w(z)+1 / w(x))^{-1}(f(z)-g(x)) \\
f(z)-\theta / w(z) \leqslant g(x)+\theta / w(x)
\end{gathered}
$$

Combining (6) and (7) we obtain

$$
\underline{h}(x) \leqslant g(x)+\theta / w(x)+\varepsilon
$$

Since $\varepsilon$ was arbitrary, we conclude that $\underline{h}(x) \leqslant g(x)+\theta / w(x)$, or

$$
w(x)(\underline{h}(x)-g(x)) \leqslant \theta .
$$

Thus (5) together with (8) imply that $\|\underline{h}-g\| \leqslant \theta$. It remains to show that $w(x)(\underline{h}(x)-f(x)) \leqslant \theta$. Indeed we have by the definition of $\theta$ together with Remark 3 that

or

$$
\begin{gathered}
\theta \geqslant \theta_{f} \geqslant(1 / w(z)+1 / w(x))^{-1}(f(z)-f(x)), \\
f(z)-\theta / w(z) \leqslant f(x)+\theta / w(x) .
\end{gathered}
$$

It follows from (6) that $\underline{h}(x) \leqslant f(x)+\theta / w(x)+\varepsilon$. Since $\varepsilon$ was arbitrary, we conclude that $\underline{h}(x) \leqslant f(x)+\theta / w(x)$, or

$$
w(x)(\underline{h}(x)-f(x)) \leqslant \theta .
$$

Combining (4),(5),(8) and (9) shows that

$$
\theta \geqslant \max (\|f-\underline{h}\|,\|g-\underline{h}\|)
$$

This establishes the main part of the theorem. The inequality is obtained by putting $h \equiv 0 \in M$.

Remark 4. In light of Theorem 2, we see that in order to exclude the case given by Remark 1(ii) we can not have $\max g \leqslant \max f$ and $\min f \leqslant \min g$ where both of $f$ and $g$ are continuous on $[a, b]$. 
Theorem 3. (Characterisation). Let $\underline{h}, \bar{h}, \theta$ and $d$ be as defined earlier. Then $\underline{h}, \bar{h} \in M, \underline{h} \leqslant \bar{h}$ and $\theta=d=\max (\|f-\underline{h}\|,\|g-\underline{h}\|)=\max (\|f-\bar{h}\|,\|g-\bar{h}\|)$. Furthermore, for $h^{*} \in M$

$$
\theta=d=\max \left(\left\|f-h^{*}\right\|,\left\|g-h^{*}\right\|\right)
$$

holds if and only if $\underline{h} \leqslant h^{*} \leqslant \bar{h}$.

Proof: By Remark 2(iii) we have $\underline{h}, \bar{h} \in M$. By Theorem 2 and a similar argument for $\bar{h}$ we obtain

$$
\theta=d=\max (\|f-\underline{h}\|,\|g-\underline{h}\|)=\max (\|f-\bar{h}\|,\|g-\bar{h}\|)
$$

Suppose now that $h^{*} \in M$ and $\theta=\max \left(\left\|f-h^{*}\right\|,\left\|g-h^{*}\right\|\right)=d$. Let $x \in[a, b]$ be arbitrary but fixed, and let $\varepsilon>0$ be given. By the definition of $\underline{h}$, there is $z \in[a, x]$ such that $\underline{h}(x) \leqslant f(z) \vee g(z)-\theta / w(z)+\varepsilon$. But

$$
\theta \geqslant \max \left(w(z)\left(f(z)-h^{*}(z)\right), w(z)\left(g(z)-h^{*}(z)\right)\right),
$$

which implies that

$$
\theta / w(z) \geqslant f(z)-h^{*}(z), \text { and } \theta / w(z) \geqslant g(z)-h^{*}(z) .
$$

Hence,

$$
h^{*}(z) \geqslant f(z) \vee g(z)-\theta / w(z) .
$$

Thus $\underline{h}(x) \leqslant h^{*}(z)+\varepsilon \leqslant h^{*}(x)+\varepsilon$. Since $\varepsilon$ was arbitrary we get $\underline{h}(x) \leqslant h^{*}(x)$. Letting $h^{*}=\bar{h}$ we end up with $\underline{h} \leqslant \bar{h}$. Similarly we show $h^{*} \leqslant \bar{h}$.

Next, let $\underline{h} \leqslant h^{*} \leqslant \bar{h}$. We show that $\max \left(\left\|f-h^{*}\right\|,\left\|g-h^{*}\right\|\right)=\theta$. Let $x \in X$.

Then

$$
\begin{aligned}
\theta & \geqslant \max (w(x)(f(x)-\underline{h}(x)), w(x)(g(x)-\underline{h}(x))), \\
& \geqslant \max \left(w(x)\left(f(x)-h^{*}(x)\right),\left(w(x)\left(g(x)-h^{*}(x)\right)\right)\right) .
\end{aligned}
$$

Also

$$
\theta \geqslant \max (w(x)(\bar{h}(x)-f(x)), w(x)(\bar{h}(x)-g(x)))
$$

$$
\geqslant \max \left(w(x)\left(h^{*}(x)-f(x)\right), w(x)\left(h^{*}(x)-g(x)\right)\right) \text {. }
$$

This says that

$$
-\theta \leqslant w(x)\left(f(x)-h^{*}(x)\right) \leqslant \theta,
$$

and similarly

$$
-\theta \leqslant w(x)\left(g(x)-h^{*}(x)\right) \leqslant \theta .
$$

Hence

$$
\theta \geqslant \max \left(\left\|f-h^{*}\right\|,\left\|g-h^{*}\right\|\right) \text {. }
$$

Equality follows from Theorem 2.

Lemma 4. Suppose $f, g$ and $w$ are continuous. Then $\underline{h}$ and $\bar{h}$ are both continuous. 
Proof: By the definition of $\underline{h}$ we may write for $y>x$,

Hence

$$
\underline{h}(y)=\max \left\{\underline{h}(x), \max _{z \in[x, y]}(f(z) \vee g(z)-\theta / w(z))\right\} .
$$

$$
\underline{h}(y)-\underline{h}(x)=\max \left\{0, \max _{z \in[x, y]}(f(z) \vee g(z)-\theta / w(z)-\underline{h}(x))\right\} .
$$

But the fact that $\underline{h}(x) \geqslant f(x) \vee g(x)-\theta / w(x)$ implies that

$$
0 \leqslant \underline{h}(y)-\underline{h}(x) \leqslant \max \left\{0, \max _{z \in[x, y]}((f(z) \vee g(z)-\theta / w(z))-(f(x) \vee g(x)-\theta / w(x)))\right\} .
$$

Since $f$ and $g$ are both continuous, we have $f \vee g-\theta / w$ is also continuous. This establishes the continuity of $\underline{h}$. Similarly we obtain the continuity of $\bar{h}$.

THEOREM 5. Let $f, g$ and $w$ be continuous with $\theta>0$. Then

$$
\begin{aligned}
& P=\bigcup_{k=1}^{n}\left[a_{k}, b_{k}\right], \quad n \geqslant 1, \\
& a \leqslant a_{k} \leqslant b_{k} \leqslant b, \quad \text { for all } k=1, \ldots, n .
\end{aligned}
$$

For $n \geqslant 2$

$$
b_{k}<a_{k+1}, \quad k=1,2, \ldots, n-1,
$$

and

$$
\left(a_{k}, b_{k}\right) \in T, \quad \text { for all } k \text {. }
$$

Proof: Clearly $m(x, y):[a, b] \times[a, b] \mapsto R$ is a continuous function. Let

$$
\Gamma_{i}=\left\{\gamma: \gamma=m(x, y),(x, y) \in T_{i}\right\} ; \quad i=1,2
$$

Define an equivalence relation $\sim$ on $T_{i}(i=1,2)$, by $\left(x_{1}, y_{1}\right) \sim\left(x_{2}, y_{2}\right) \Longleftrightarrow m\left(x_{1}, y_{1}\right)=$ $m\left(x_{2}, y_{2}\right)$, where $\left(x_{1}, y_{1}\right),\left(x_{2}, y_{2}\right) \in T_{i}$. Then the sets

$$
\begin{aligned}
& T_{1}^{\gamma}=\left\{(x, y) \in T_{1}: m(x, y)=\gamma\right\} \\
& T_{2}^{\gamma}=\left\{(x, y) \in T_{2}: m(x, y)=\gamma\right\}
\end{aligned}
$$

are equivalence classes.

For each $\gamma \in \Gamma=\Gamma_{1} \cup \Gamma_{2}$, let

$$
\begin{array}{ll} 
& T_{\gamma}=T_{1}^{\gamma} \cup T_{2}^{\gamma} . \\
\text { Also, let } & a_{\gamma}=\inf \left\{x:(x, y) \in T_{\gamma}\right\}, \\
\text { and } & b_{\gamma}=\sup \left\{y:(x, y) \in T_{\gamma}\right\} .
\end{array}
$$

Clearly $a_{\gamma}=b_{\gamma}$ if and only if $T_{\gamma}=(x, x)$ for a single point $x \in[a, b]$. Suppose $a_{\gamma}<b_{\gamma}$. We assert that $m\left(a_{\gamma}, b_{\gamma}\right)=\gamma$, and so $\left(a_{\gamma}, b_{\gamma}\right) \in T_{\gamma}$. Indeed by the definitions 
of inf and sup, there are sequences $\left(x_{n}, y_{n}\right),\left(u_{n}, v_{n}\right) \in T, n=1,2, \ldots$ such that $x_{n} \rightarrow a_{\gamma}$ and $v_{n} \rightarrow b_{\gamma}$. Let us assume without loss of generality that $\left(x_{n}, y_{n}\right) \in T_{1}$, so we obtain for all $n$,

$$
m\left(x_{n}, y_{n}\right)=\left(w\left(x_{n}\right)+w\left(y_{n}\right)\right)^{-1}\left(w\left(x_{n}\right) f\left(x_{n}\right)+w\left(y_{n}\right) g\left(y_{n}\right)\right)=\gamma
$$

and

$$
\theta=\left(w\left(x_{n}\right)+w\left(y_{n}\right)\right)^{-1} w\left(x_{n}\right) w\left(y_{n}\right)\left(f\left(x_{n}\right)-g\left(y_{n}\right)\right)
$$

We now have two cases to consider:

Case 1. There is a subsequence $\left(u_{n}, v_{n}\right) \in T_{1}$ such that $v_{n} \rightarrow b_{\gamma}$, and

$$
m\left(u_{n}, v_{n}\right)=\left(w\left(u_{n}\right)+w\left(v_{n}\right)\right)^{-1}\left(w\left(u_{n}\right) f\left(u_{n}\right)+w\left(v_{n}\right) g\left(v_{n}\right)\right)=\gamma
$$

and,

$$
\theta=\left(w\left(u_{n}\right)+w\left(v_{n}\right)\right)^{-1} w\left(u_{n}\right) w\left(v_{n}\right)\left(f\left(u_{n}\right)-g\left(v_{n}\right)\right)
$$

Hence from (12) and (13) we get

or

$$
\begin{aligned}
\theta / w\left(x_{n}\right)+\theta / w\left(y_{n}\right) & =f\left(x_{n}\right)-g\left(y_{n}\right) \\
f\left(x_{n}\right)-\theta / w\left(x_{n}\right) & =g\left(y_{n}\right)+\theta / w\left(y_{n}\right)=\gamma .
\end{aligned}
$$

Simularly (14) and (15) imply that

Hence,

$$
\begin{aligned}
& f\left(u_{n}\right)-\theta / w\left(u_{n}\right)=g\left(v_{n}\right)+\theta / w\left(v_{n}\right)=\gamma \\
& f\left(x_{n}\right)-\theta / w\left(x_{n}\right)=\gamma=g\left(v_{n}\right)+\theta / w\left(v_{n}\right)
\end{aligned}
$$

Letting $n \rightarrow \infty$, we conclude by the continuity of $f, g$ and $w$ that

$$
f\left(a_{\gamma}\right)-\theta / w\left(a_{\gamma}\right)=\gamma=g\left(b_{\gamma}\right)+\theta / w\left(b_{\gamma}\right)
$$

so that

$$
\left(w\left(a_{\gamma}\right)+w\left(b_{\gamma}\right)\right)^{-1} w\left(a_{\gamma}\right) w\left(b_{\gamma}\right)\left(f\left(a_{\gamma}\right)-g\left(b_{\gamma}\right)\right)=\theta
$$

Thus, $\left(a_{\gamma}, b_{\gamma}\right) \in T$. Substituting for $\theta$ in (17), using the first part of (16), we conclude that $m\left(a_{\gamma}, b_{\gamma}\right)=\gamma$. This proves the assertion for case 1 . 
Case 2. There is no sequence $\left(u_{n}, v_{n}\right) \in T_{1}^{\gamma}$ for which $v_{n} \rightarrow b_{\gamma}$, that is, $v_{n} \rightarrow b_{\gamma}$ if and only if $\left(u_{n}, v_{n}\right) \in T_{2}^{\gamma}$. In such a case we can argue that $\theta=\theta_{f}$ which is contradictory to our assumption. Therefore only case 1 is valid.

Next we show that for $(x, y) \in T, x<y$ we have $[x, y] \cap\left[a_{\gamma}, b_{\gamma}\right] \neq \emptyset$ if and only if $m(x, y)=\gamma$. By the definition of $\theta$, we have

$$
f(x)-\theta / w(x) \leqslant g(y)+\theta / w(y)
$$

If $\left[a_{\gamma}, b_{\gamma}\right] \cap[x, y] \neq \emptyset$, then it follows from the definition of $a_{\gamma}$ and $b_{\gamma}$ that $a_{\gamma} \leqslant y$ and $b_{\gamma} \geqslant x$. From $(16),(17),(18)$ and the definition of $\theta$ it follows that

$$
\begin{aligned}
f(x)-\theta / w(x) & \leqslant g\left(b_{\gamma}\right)+\theta / w\left(b_{\gamma}\right)=\gamma \\
& =f\left(a_{\gamma}\right)-\theta / w\left(a_{\gamma}\right) \\
& \leqslant g(y)+\theta / w(y) .
\end{aligned}
$$

Since $(x, y) \in T,(18)$ holds with equality, and therefore (19) implies that

$$
f(x)-\theta / w(x)=g(y)+\theta / w(y)=\gamma,
$$

or alternatively $m(x, y)=\gamma$. The converse follows immediately from the definition of $a_{\gamma}$ and $b_{\gamma}$.

By the uniform continuity of $f$ and $g$ we can easily deduce the first part of the theorem, that is, $\Gamma$ is finite and hence $P$ is a finite union of closed sub-intervals.

Theorem 6. Let $f, g, w, \theta$ and $P$ be as in the previous Theorem. Then

$$
\underline{h}(x)=\bar{h}(x) \text { if and only if } x \in P,
$$

with

$$
\underline{h}(x)=\bar{h}(x)=m\left(a_{k}, b_{k}\right) \text { for all } x \in\left[a_{k}, b_{k}\right] \text { and all } k \text {, }
$$

where

$$
m\left(a_{k}, b_{k}\right)<m\left(a_{k+1}, b_{k+1}\right), \quad k=1,2, \ldots, n-1 \text {. }
$$

Moreover

$$
w(x)|f(x)-\underline{h}(x)|=w(y)|g(y)-\bar{h}(y)|=\theta, \quad(x, y) \in T_{1},
$$

and

$$
w(y)|f(y)-\bar{h}(y)|=w(x)|g(x)-\underline{h}(x)|=\theta, \quad(x, y) \in T_{2} .
$$

Proof: To obtain the first part of the Theorem we start by showing that $\underline{h}(x)=\gamma_{k}$ for all $x \in\left[a_{k}, b_{k}\right]$. In (16), let $a_{\gamma}=a_{k}, b_{\gamma}=b_{k}$ and $\gamma=\gamma_{k}$. Hence we obtain

$$
f\left(a_{k}\right)-\theta / w\left(a_{k}\right)=\gamma_{k}=g\left(b_{k}\right)+\theta / w\left(b_{k}\right), \quad k=1, \ldots, n .
$$

If $a \leqslant z \leqslant b_{k}$, then by the definition of $\theta$ we have

$$
f(z)-\theta / w(z) \leqslant g\left(b_{k}\right)+\theta / w\left(b_{k}\right)=\gamma_{k} .
$$


Since $\theta \geqslant \theta_{g}$, it follows that

and hence

$$
\begin{gathered}
g(z)-\theta / w(z) \leqslant g\left(b_{k}\right)+\theta / w\left(b_{k}\right)=\gamma_{k}, \\
f(z) \vee g(z)-\theta / w(z) \leqslant \gamma_{k} .
\end{gathered}
$$

From the definition of $\underline{h}$ we conclude that $\underline{h}(x) \leqslant \gamma_{k}$ for all $x \in\left[a, b_{k}\right]$. But then

$$
\underline{h}\left(a_{k}\right) \geqslant f\left(a_{k}\right)-\theta / w\left(a_{k}\right)=\gamma_{k} .
$$

By monotonicity of $\underline{h}$ it follows that for any $x \in\left[a_{k}, b_{k}\right]$ we have

$$
\underline{h}(x) \geqslant \underline{h}\left(a_{k}\right) \geqslant \gamma_{k} \text {. }
$$

Hence $\underline{h}(x)=\gamma_{k}$ for all $x \in\left[a_{k}, b_{k}\right]$.

Similarly we show that $\bar{h}(x)=\gamma_{k}$ for all $x \in\left[a_{k}, b_{k}\right]$.

We now prove the second part of the theorem consisting of the last two equations . Let $(x, y) \in T=T_{1} \cup T_{2}$. Assume without loss of generality that $(x, y) \in T_{1}$. The other case is similar. Since $\Gamma$ is finite, we must have $m(x, y)=\gamma_{k}$ for some $k=1,2, \ldots, n$, so it follows that $[x, y] \subseteq\left[a_{k}, b_{k}\right]$. Since $\underline{h}$ is non-decreasing we have

$$
\gamma_{k}=\underline{h}\left(a_{k}\right) \leqslant \underline{h}(x) \leqslant \underline{h}(y) \leqslant \underline{h}\left(b_{k}\right)=\gamma_{k},
$$

so that $\underline{h}(x)=\gamma_{k}$ and

$$
\begin{aligned}
w(x)|f(x)-\underline{h}(x)| & =w(x)|f(x)-m(x, y)| \\
& =w(x)\left|f(x)-(w(x)+w(y))^{-1}(w(x) f(x)+w(y) g(y))\right| \\
& =(w(x)+w(y))^{-1} w(x) w(y)|f(x)-g(y)|=\theta
\end{aligned}
$$

Similarly,

$$
\begin{aligned}
w(y)|g(y)-\bar{h}(y)| & =w(y)|g(y)-m(x, y)| \\
& =w(y)\left|g(y)-(w(x)+w(y))^{-1}(w(x) f(x)+w(y) g(y))\right| \\
& =(w(x)+w(y))^{-1} w(x) w(y)|g(y)-f(x)|=\theta
\end{aligned}
$$

LEMMA 7. Suppose that $f, g$ and $w$ are continuous, and that $0<\theta_{f}, \theta_{g}<\theta$.

Then

and

$$
\begin{array}{ll}
\bar{h}(x)>\gamma_{k}, & \text { for } x>b_{k}, \\
\underline{h}(x)<\gamma_{k}, & \text { for } x<a_{k} .
\end{array}
$$

Proof: Suppose that for some $x>b_{k}$ we have $\bar{h}(x)=\gamma_{k}$. Then by the definition of $\bar{h}(x)$, there exists $y \in[x, b]$ such that

$$
\bar{h}(x)=\gamma_{k}=\min (f(y), g(y))+\theta / w(y) .
$$

We have two cases to consider: 
Case 1. $f(y)<g(y)$, so that

$$
\bar{h}(x)=f(y)+\theta / w(y)=\gamma_{k} .
$$

In such a case we claim that $\theta=u\left(a_{k}, b_{k}\right)\left(g\left(a_{k}\right)-f\left(b_{k}\right)\right)$. If not, then we must have $\theta=u\left(a_{k}, b_{k}\right)\left(f\left(a_{k}\right)-g\left(b_{k}\right)\right)$. Hence

$$
f\left(a_{k}\right)-\theta / w\left(a_{k}\right)=f\left(a_{k}\right)-u\left(a_{k}, b_{k}\right)\left(f\left(a_{k}\right)-g\left(b_{k}\right)\right) / w\left(a_{k}\right)=\gamma_{k} .
$$

Combining (20) and (21) results in

or

$$
\begin{gathered}
f\left(a_{k}\right)-\theta / w\left(a_{k}\right)=f(y)+\theta / w(y), \\
\theta=\frac{w\left(a_{k}\right) w(y)}{\left(w\left(a_{k}\right)+w(y)\right)}\left(f\left(a_{k}\right)-f(y)\right) \leqslant \theta_{f} .
\end{gathered}
$$

This is a contradiction! Therefore our clain holds and we have

$$
\begin{array}{ll}
\qquad \begin{aligned}
g\left(a_{k}\right)-\theta / w\left(a_{k}\right)= & g\left(a_{k}\right)-\left(w\left(a_{k}\right)+w\left(b_{k}\right)\right)^{-1} w\left(b_{k}\right)\left(g\left(a_{k}\right)-f\left(b_{k}\right)\right), \\
= & \left(w\left(a_{k}\right)+w\left(b_{k}\right)\right)^{-1}\left(w\left(a_{k}\right) g\left(a_{k}\right)+w\left(b_{k}\right) f\left(b_{k}\right)\right), \\
= & \gamma_{k}=f(y)+\theta / w(y),
\end{aligned} \\
\text { or } \quad g\left(a_{k}\right)-f(y)=\left(1 / w\left(a_{k}\right)+1 / w(y)\right) \theta . \\
\text { Hence } & \theta=u\left(a_{k}, y\right)\left(g\left(a_{k}\right)-f(y)\right) .
\end{array}
$$

which implies that $\left(a_{k}, y\right) \in T$, and by Theorem 5 we conclude that $\left[a_{k}, y\right] \subseteq\left[a_{k}, b_{k}\right]$ which is a contradiction since $a_{k} \leqslant b_{k}<y$. Therefore there is no $x>b_{k}$, for which $\bar{h}(x)=\gamma_{k}$, that is, $\bar{h}(x)>\gamma_{k}$ for $x>b_{k}$.

Case 2. $g(y)<f(y)$. The same argument applies. This concludes the proof of the first part. The other part is similar.

THEOREM 8. Let $f, g$ and $w$ be continuous on $[a, b]$. If $0<\theta_{f}, \theta_{g}<\theta$, then

$$
\underline{h}(x)<\bar{h}(x) \text { for all } x \in\left[a, a_{1}\right] \cup\left(\bigcup_{k=1}^{n-1}\right) \cup\left(b_{n}, b\right] \text {. }
$$

Proof: Suppose that for some $t \in\left(b_{k}, a_{k+1}\right), k=1,2, \ldots, n-1$, we have $\underline{h}(t)=\bar{h}(t)$. Then by the definitions of $\underline{h}$ and $\bar{h}$, there exists $u \in\left[b_{k}, t\right], v \in\left[t, a_{k+1}\right]$ such that

$$
\begin{aligned}
\underline{h}(t) & =\max (f(u), g(u))-\theta / w(u), \\
& =\min (f(v), g(v))+\theta / w(v)=\bar{h}(t) .
\end{aligned}
$$

Notice that if $u<b_{k}$, then clearly $\underline{h}(x)=\underline{h}\left(b_{k}\right)=\gamma_{k}$ for all $x \in\left(b_{k}, t\right]$ which contradicts the definition of $b_{k}$. Therefore $u \in\left[b_{k}, t\right]$. Similarly we must have $v \in$ $\left[t, a_{k+1}\right]$. In (22) suppose $f(u)>g(u)$. We also have two cases here: 
Case 1. $f(v)>g(v)$, so we obtain

$$
\underline{h}(t)=f(u)-\theta / w(u)=g(v)+\theta / w(v)=\bar{h}(t),
$$

or

$$
\theta=\frac{w(u) w(v)}{(w(u)+w(v))}(f(u)-g(v)), \quad u<v .
$$

This says that $(u, v) \in T$, or there exists some $i$ such that $(u, v) \subseteq\left[a_{i}, b_{i}\right]$. This is a contradiction, since we have $b_{k} \leqslant u \leqslant t \leqslant v \leqslant a_{k+1}$.

Case 2. $f(v)<g(v)$, so we obtain

or

$$
\underline{h}(t)=f(u)-\theta / w(u)=f(v)+\theta / w(v)=\bar{h}(t),
$$

$$
\theta=\frac{w(u) w(v)}{(w(u)+w(v))}(f(u)-f(v)) \leqslant \theta_{f},
$$

contradicting our assumption that $\theta>\theta_{f}$.

In the cases $t \in\left[a, a_{1}\right]$ or $t \in\left(b_{n}, b\right]$ we follow the same line of argument. Hence, Theorem 8.

\section{REFERENCES}

[1] V.A. Ubhaya, 'Isotone optimization I', J. Approx. Theory 12 (1974), 146-159.

\section{Department of Mathematics}

Faculty of Science, KAU

P.O. Box 9028

Jeddah 21413

Saudi Arabia. 